\title{
SpRY greatly expands the genome editing scope in rice with highly flexible PAM recognition
}

\author{
Ziyan Xu ${ }^{1,2+}$, Yongjie Kuang ${ }^{1,2+}$, Bin Ren ${ }^{1,2,3 \dagger}$, Daqi Yan ${ }^{1,4}$, Fang Yan ${ }^{1}$, Carl Spetz ${ }^{5}$, Wenxian Sun ${ }^{4,6}$, Guirong Wang ${ }^{1,3}$, \\ Xueping Zhou ${ }^{1,7^{*}}$ and Huanbin Zhou ${ }^{1,2^{*}}$ (i)
}

\author{
* Correspondence: zzhou@zju.edu. \\ cn; hbzhou@ippcaas.cn \\ 'Ziyan Xu, Yongjie Kuang and Bin \\ Ren contributed equally to this \\ work. \\ ${ }^{1}$ State Key Laboratory for Biology of \\ Plant Diseases and Insect Pests, \\ Institute of Plant Protection, \\ Chinese Academy of Agricultural \\ Sciences, Beijing 100193, China \\ Full list of author information is \\ available at the end of the article
}

\begin{abstract}
Background: Plant genome engineering mediated by various CRISPR-based tools requires specific protospacer adjacent motifs (PAMs), such as the well-performed $N G G, N G$, and NNG, to initiate target recognition, which notably restricts the editable range of the plant genome.

Results: In this study, we thoroughly investigate the nuclease activity and the PAM preference of two structurally engineered SpCas9 variants, SpG and SpRY, in transgenic rice. Our study shows that SpG nuclease favors NGD PAMs, albeit less efficiently than the previously described SpCas9-NG, and that SpRY nuclease achieves efficient editing across a wide range of genomic loci, exhibiting a preference of NGD as well as NAN PAMs. Furthermore, SpRY-fused cytidine deaminase hAID* $\Delta$ and adenosine deaminase TadA8e are generated, respectively. These constructs efficiently induce C-to-T and A-to-G conversions in the target genes toward various noncanonical PAMs, including non-G PAMs. Remarkably, high-frequency self-editing events (indels and DNA fragments deletion) in the integrated T-DNA fragments as a result of the nuclease activity of SpRY are observed, whereas the self-editing of SpRY nickase-mediated base editor is quite low in transgenic rice lines.
\end{abstract}

Conclusions: The broad PAM compatibility of SpRY greatly expands the targeting scope of CRISPR-based tools in plant genome engineering.

Keywords: CRISPR, SpG, SpRY, Genome editing, Base editing, Self-targeting, Oryza sativa L.

\section{Background}

CRISPR technologies, which enable a variety of precise genetic modifications through targeted genome editing across nearly all living organisms, have been intensively developed in recent years and revolutionized agricultural studies. CRISPR is derived from the defense system of bacteria and archaea, protecting them from the invasion of bacteriophages and mobile genetic elements [1]. A large collection of CRISPR/Cas systems have been identified by genome sequencing and metagenome studies. Among these, the type II CRISPR/SpCas9 from Streptococcus pyogenes has been extensively studied

(c) The Author(s). 2021 Open Access This article is licensed under a Creative Commons Attribution 4.0 International License, which permits use, sharing, adaptation, distribution and reproduction in any medium or format, as long as you give appropriate credit to the original author(s) and the source, provide a link to the Creative Commons licence, and indicate if changes were made. The images or other third party material in this article are included in the article's Creative Commons licence, unless indicated otherwise in a credit line to the material. If material is not included in the article's Creative Commons licence and your intended use is not permitted by statutory regulation or exceeds the permitted use, you will need to obtain permission directly from the copyright holder. To view a copy of this licence, visit http://creativecommons.org/licenses/by/4.0/. The Creative Commons Public Domain Dedication waiver (http://creativecommons.org/publicdomain/zero/1.0/) applies to the data made available in this article, unless otherwise stated in a credit line to the data. 
and adapted for genome editing. For example, SpCas9 nuclease can be used to genetically modify a specific target locus within the plant genome. Briefly, SpCas9 catalyzes a DNA double-stranded break (DSB) which subsequently stimulates diverse DNA repair mechanisms [non-homologous end joining (NHEJ), microhomology-mediated end joining (MMEJ), and homology-directed repairs (HDR)] resulting in gene knockout, DNA fragment insertion, deletion, and replacement as specifically required [2-6]. Furthermore, the SpCas9(D10A) nickase has been utilized to drive various engineered cytine deaminases (e.g., rAPOBEC1, hAID* $\Delta$, hAPOBEC3A) to the target region for cytosine base editing where it catalyzes the hydrolytic deamination of cytosines to uracils within the editing window [7-11]. As a result, the mismatched DNA base pairs are processed through the base excision repair pathway, leading to various base transitions and transversions: C-to-D (where D is T, G, or A) and G-to-H (where H is A, C, or T). Alternatively, the SpCas9(D10A) nickase is also compatible with numerous adenosine deaminase (i.e., E. coli TadA variants TadA7.10, TadA8.20, TadA8e) for adenine base editing [12-14], which turn adenines to inosines in the target genomic DNA region, resulting in pure A-to-G and T-to-C conversions. In addition, the dual base editors, constructed by fusing both cytidine deaminase and adenosine deaminase to the SpCas9(D10A) nickase, have been developed and are capable of concurrently editing C and A [15-17]. Recently, the SpCas9(H840A) nickase-guided Moloney murine leukemia virus reverse transcriptase (M-MLV RT) has been developed in both rice and wheat, facilitating the introduction of all 12 base-to-base conversions, small insertion, and deletions in a precise and targeted manner through reverse transcription of the pegRNA [18-21]. Thus, diverse customized CRISPR/SpCas9 tools for precise and versatile genome editing greatly accelerate our understanding of the genetic basis of economic traits and the generation of novel germplasms for crop breeding.

It is well known that SpCas9 recognizes the canonical NGG PAM which is located immediately downstream of the target sequence [22]. Initial NGG PAM binding by SpCas9 triggers DNA strand separation locally and facilitates base pairing between spacer RNA and the target DNA strand [23]. In other words, the presence of a canonical NGG PAM near the target sites is critical for CRISPR/SpCas9-mediated genome editing. SpCas 9 and its related tools have been shown to perform efficiently in genetic manipulation of a wide range of plant species [2, 9, 24-27]. However, the specific PAM requirement for SpCas9 recognition strongly restricts the targetable loci of the CRIS $\mathrm{PR} / \mathrm{SpCas} 9$-based editing tools, especially base editors because the targeted point mutation needs the availability of a PAM appropriately positioned [7, 8, 12, 28].

To expand the genome-targeting scope of CRISPR tools, many efforts have been focused on searching for new Cas variants and orthologues with novel PAM preferences. For example, directed evolution and structure-guided design of SpCas9 resulted in SpCas9-VRQR, xCas9, and Cas9-NG variants that recognize non-canonical NGA and NG PAM sites in plant [29-32]. Multiple naturally occurring SpCas9 orthologues have been identified from Streptococcus canis (ScCas9), Staphylococcus aureus (SaCas9), Streptococcus thermophiles (St1Cas9), and Brevibacillus laterosporus (BlatCas9) and have been demonstrated to edit plant genomic loci bearing NNG, NNGRRT, NNAG AAW, and NNNCND PAM sequence, respectively [33-36]. Also, the type V Cas12a and Cas12b from diverse bacterial sources, which are distinct from Cas9, have been characterized with AT-rich PAM specificity and utilized successfully in targeted plant 
genome editing [37, 38]. Regardless of the fact that these Cas proteins with different PAM sequences significantly improve the targeting range, there are still many agronomic trait-related loci inaccessible for genome editing.

Very recently, two variants (SpG and SpRY) engineered from SpCas9-VRQR through structure-guided design have been reported to recognize a wider range of PAM sequences. SpG has higher genome-editing activity toward NG PAM than SpCas9-NG, whereas SpRY is capable of targeting almost all PAMs (NRN>NYN) [39]. Both SpG and SpRY exhibit robust activities with minimal side effects on a wide range of sites in human cells [39]. Furthermore, an evolved adenosine deaminase TadA8e, which catalyzes DNA deamination up to $\sim 1100$-fold faster than the previous version TadA7.10, has been reported to substantially improve adenine base editing in human cells $[14,40]$. However, whether SpG, SpRY, and TadA8e can be utilized to improve genome editing, especially base editing, in plants remains unknown. In this study, the efficiency of both SpG and SpRY nucleases toward various PAMs and their application in both cytosine and adenine base editing was investigated in detail using transgenic rice callus. Our study shows that SpG recognizes NG PAM sequences, but it is outperformed by SpCas9-NG in rice. However, albeit with self-targeting activity on transfer T-DNA sequence, SpRY surpasses SpCas9-NG since it is capable of achieving efficient cleavage by its nuclease activity, cytosine base editing with hAID* $\Delta$, and adenine base editing with TadA8e at more relaxed PAM sites (NRN, where $R$ is G or A) in rice. Therefore, SpRY and TadA8e facilitate the future design of genome editing tools in plants and broaden the possible applications in agriculture and plant biology.

\section{Results}

SpCas9-NG nuclease outperforms SpG on the minimal NG PAM in transgenic rice lines We first compared the DNA-cleavage capability and the PAM specificity of SpG nuclease to that of SpCas9-NG (which is well known for NG PAM recognition) in targeted genome editing in transgenic rice. SpCas9 carrying mutations D1135L/S1136W/ G1218K/E1219Q/R1335Q/T1337R was rice codon-optimized (Additional file 1: Table S1) and used to replace the SpCas9-NG gene in the binary vector pUbi:SpCas9-NG [32], resulting in pUbi:SpG in which $S p G$ is under the control of the maize ubiquitin 1 promoter (Fig. 1a). The rice U6 promoter-driven sgRNA expression cassette can be shuttled into both binary vectors through Gateway recombination reaction as previously described [32]. The nuclease activity of SpG and SpCas9-NG was tested side-by-side with the same sgRNAs targeting the endogenous OsPAL5, OsGSK4, OsCERK1, OsETR2, and OsRLCK185 genes toward four types of NGN PAMs through Agrobacterium-mediated rice transformation, respectively (Additional file 1: Figure S1; S2). Target regions in T0 independent transgenic callus lines were PCR amplified and directly subjected to Sanger sequencing.

Analysis of 40 independent lines for each NGG PAM site tested with SpG nuclease revealed twelve lines that carried indel mutations in OsPAL5 and four mutant lines of OsGSK4, representing 30\% and 10\% editing efficiency, respectively (Fig. 1b, c). By contrast, SpCas9-NG achieved 62.50\% (25 out of 40 lines) editing efficiency in OsPAL5, a 1.1-fold increase over that of SpG, and 20.51\% (8 out of 39 lines) editing efficiency in OsGSK4, a 1.1-fold increase over SpG (Fig. 1b, c). At the NGA PAM site tested, SpG generated much fewer indel mutations $(<4$-fold) than SpCas9-NG, with $11.11 \%$ of lines 
a

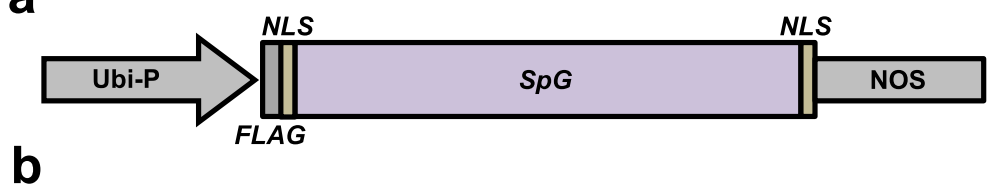

\begin{tabular}{|c|c|c|c|c|c|}
\hline Locus & $\begin{array}{l}\text { PAM } \\
\text { seq }\end{array}$ & Nuclease & $\begin{array}{l}\text { Mutation } \\
\text { efficiency }\end{array}$ & $\begin{array}{c}\text { Mono-allelic } \\
\text { mutation }\end{array}$ & $\begin{array}{l}\text { Bi-allelic } \\
\text { mutation }\end{array}$ \\
\hline \multirow{2}{*}{$\begin{array}{l}\text { OsPAL5 } \\
\text { spacer } 1\end{array}$} & \multirow{2}{*}{ NGG } & SpCas9-NG & $25 / 40(62.50 \%)$ & $11 / 25$ & $14 / 25$ \\
\hline & & SpG & $12 / 40(30.00 \%)$ & $8 / 12$ & $4 / 12$ \\
\hline \multirow{2}{*}{$\begin{array}{l}\text { OsGSK4 } \\
\text { spacer } 1\end{array}$} & \multirow{2}{*}{ NGG } & SpCas9-NG & $8 / 39(20.51 \%)$ & $8 / 8$ & $0 / 8$ \\
\hline & & SpG & $4 / 40(10.00 \%)$ & $4 / 4$ & $0 / 4$ \\
\hline \multirow{2}{*}{$\begin{array}{c}\text { OsCERK1 } \\
\text { spacer } 1\end{array}$} & \multirow{2}{*}{ NGA } & SpCas9-NG & $19 / 41(46.34 \%)$ & $5 / 19$ & $14 / 19$ \\
\hline & & SpG & $5 / 45(11.11 \%)$ & $3 / 5$ & $2 / 5$ \\
\hline \multirow{2}{*}{$\begin{array}{l}\text { OsGSK4 } \\
\text { spacer } 2\end{array}$} & \multirow{2}{*}{ NGT } & SpCas9-NG & $20 / 38(52.63 \%)$ & $16 / 20$ & $4 / 20$ \\
\hline & & SpG & $17 / 47(36.17 \%)$ & $14 / 17$ & $3 / 17$ \\
\hline \multirow{2}{*}{$\begin{array}{l}\text { OsETR2 } \\
\text { spacer } 1\end{array}$} & \multirow{2}{*}{ NGT } & SpCas9-NG & $2 / 38(5.26 \%)$ & $2 / 2$ & $0 / 2$ \\
\hline & & SpG & $1 / 45(2.22 \%)$ & $1 / 1$ & $0 / 1$ \\
\hline \multirow{2}{*}{$\begin{array}{l}\text { OsCERK1 } \\
\text { spacer } 2\end{array}$} & \multirow{2}{*}{ NGC } & SpCas9-NG & $5 / 35(14.29 \%)$ & $5 / 5$ & $0 / 5$ \\
\hline & & SpG & $5 / 32(15.63 \%)$ & $4 / 5$ & $1 / 5$ \\
\hline \multirow{2}{*}{$\begin{array}{c}\text { OsRLCK185 } \\
\text { spacer } 1\end{array}$} & \multirow{2}{*}{ NGC } & SpCas9-NG & $1 / 39(2.56 \%)$ & $1 / 1$ & $0 / 1$ \\
\hline & & SpG & $1 / 48(2.08 \%)$ & $1 / 1$ & $0 / 1$ \\
\hline
\end{tabular}

C

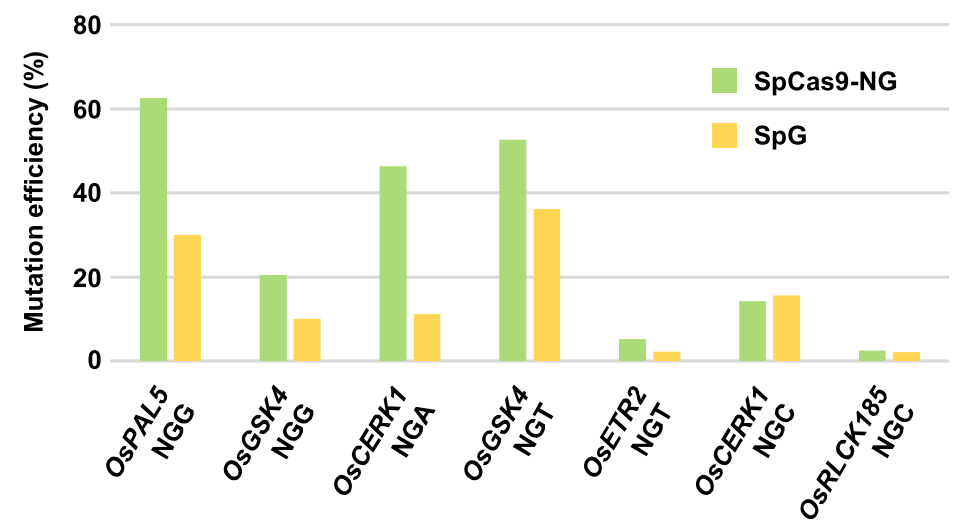

Fig. 1 Analysis of SpG nuclease activity on different NGN PAMs in transgenic rice. a The gene construct of SpG nuclease used for genome editing in transgenic rice. Ubi-P, maize ubiquitin 1 promoter; NLS, nuclear localization signal. b Summary of the genotyping results on T0 transgenic rice lines generated with SpG and SpCas9-NG nuclease. c Comparison of the mutation frequency by SpG and SpCas9-NG nucleases toward 4 NGN PAMs in TO transgenic rice lines

edited by SpG carried indels in OsCERK1 as compared to $46.34 \%$ of the lines edited by SpCas9-NG (Fig. 1b, c). At the NGT PAM sites tested, the editing efficiency of SpG was $36.17 \%$ (17 out of 47 lines) in OsGSK4 and 2.22\% (1 out of 45 lines) in OsETR2, whereas the editing efficiency of SpCas9-NG was higher, being $52.63 \%$ and $5.26 \%$ in OsGSK4 and in OsETR2, respectively (Fig. 1b, c). In the case of the NGC PAM, which has been reported to be less efficiently recognized by SpCas9-NG [32, 34], SpG edited 
OsCERK1 and OsRLCK185 at the corresponding sites with comparable efficiency to SpCas9-NG (Fig. 1b, c). It should be noted that both bi-allelic and mono-allelic mutations were identified in T0 mutant lines generated by SpG toward all PAM sequences, with a relatively higher rate of mono-allelic edits (Fig. 1b, c; Additional file 1: Figures S1; S2). Overall, these results indicate that the newly engineered SpG nuclease is capable of recognizing minimal NG PAM in rice, albeit less efficiently than SpCas9-NG. Consequently, the activity of $\mathrm{SpG}$ in plants is different from the previously reported SpG-mediated genome editing efficiency in human cells [39].

SpRY nuclease preferentially recognizes NAN and NGN PAM sequences in transgenic rice lines Five other point mutations consisting of A61R, L1111R, N1317R, A1322R, and R1333P were further introduced into SpG to generate SpRY (Additional file 1: Table S1; Figure S3a). Following the same experimental procedure mentioned above, the nuclease activity and PAM preference of SpRY were thoroughly investigated in transgenic rice calli. Thirty-two endogenous genomic sites bearing all 16 possible alternative PAM sequences, which vary at the second and the third positions, were used (Additional file 1: Figures S3-S16). Each transformation construct used harbored two sgRNAs targeting the same gene or different genes to the extent that multiplex genome editing and/or DNA fragment deletion could be achieved and investigated in rice cells.

Genotyping of all the T0 transgenic lines for the NGN PAM sites showed that SpRY induces indel mutations predominantly at the NGG PAM site $(63.83 \%$ editing), followed by the NGA (>74\% editing) and NGT (>47\% editing) PAM sites. The NGC PAM site is the least recognized, achieving only a $2.13 \%$ editing efficiency (Fig. 2a, b). Thus, SpRY nuclease favors the recognition of NGD $>$ NGC PAM in rice. On the NAN PAM sites tested, the editing efficiency of SpRY was highly variable, but it reached as high as $20.00 \%$ for a NAG PAM site in OsMPK8, $33.33 \%$ for a NAA site in OsMPK9, $51.28 \%$ for a NAT site in OsMPK10, and 29.79\% for a NAC site in OsCPK2 (Fig. 2a, b), suggesting that SpRY moderately recognizes NAN PAM sequences in rice, albeit in a locus-dependent manner. Across the rest 16 genomic sites with NYN PAMs, SpRY exhibited editing at 4 sites (25\%), with a high activity on a NTC PAM site in OsCPK28 $(73.91 \%$ efficiency) and weak activity on a NCG site in OsCPK20 (2.94\% editing), a NCT site in OsMPK3 (4.26\% editing), and a NCC site in OsMPK4 (7.69\% editing) (Fig. 2a, b). It should be mentioned that screening of all target genes revealed that the majority of edited lines contained mono-allelic mutations at the target sites, and that only 2 out of 47 independent lines (4.26\% ratio) had genomic DNA fragment deletions in OsCPK2 (Fig. 2a; Additional file 1: Figures S3-S16). Combining all the data (Fig. 2b), we conclude that the structurally engineered SpRY is capable of recognizing NRN and some NYN PAMs in rice, but with impaired nuclease activity as compared to that of SpCas9 reported previously [2].

The broad PAM compatibility of SpRY nuclease implies that SpRY might be similar to SpCas9-NG which was reported to self-edit the single-guide RNA region of the transferred T-DNA in transgenic rice cells [41]. Therefore, the identities of the sgRNA transgenes in some $S p R Y$-transgenic lines were examined by direct PCR sequencing. For the high-efficiency OsCPK5-sgRNA1, OsCPK1-sgRNA1, and OsCPK1-sgRNA2, no 


\begin{tabular}{|c|c|c|c|c|c|c|c|c|c|}
\hline Construct & $\begin{array}{c}\text { sgRNA } \\
\text { type }\end{array}$ & Spacer & $\begin{array}{c}\text { PAM } \\
\text { seq }\end{array}$ & $\begin{array}{c}\text { Mutation } \\
\text { efficiency }\end{array}$ & $\begin{array}{c}\text { Mono-allelic } \\
\text { mutation }\end{array}$ & $\begin{array}{l}\text { Bi-allelic } \\
\text { mutation }\end{array}$ & $\begin{array}{l}\text { Self-editing } \\
\text { frequency }\end{array}$ & $\begin{array}{c}\text { Spacer } \\
\text { distance }\end{array}$ & $\begin{array}{c}\text { Deletion } \\
\text { ratio }\end{array}$ \\
\hline \multirow{2}{*}{ OsCPK5 } & \multirow{2}{*}{ GTTT } & 1 & NGG & $30 / 47(63.83 \%)$ & $25 / 30(83.33 \%)$ & $5 / 30(16.67 \%)$ & $2 / 47(4.26 \%)$ & \multirow{2}{*}{$216 \mathrm{bp}$} & \multirow{2}{*}{0} \\
\hline & & 2 & NGG & $0 / 47(0.00 \%)$ & $0 / 0(0.00 \%)$ & $0 / 0(0.00 \%)$ & $0 / 47(0.00 \%)$ & & \\
\hline \multirow{2}{*}{ OsCPK1 } & \multirow{2}{*}{ GTTT } & 1 & NGA & $18 / 31(58.06 \%)$ & $18 / 18(100.00 \%)$ & $0 / 18(0.00 \%)$ & $0 / 31(0.00 \%)$ & \multirow{2}{*}{$207 \mathrm{bp}$} & \multirow{2}{*}{0} \\
\hline & & 2 & NGA & $23 / 31(74.19 \%)$ & $23 / 23(100.00 \%)$ & $0 / 23(0.00 \%)$ & $0 / 31(0.00 \%)$ & & \\
\hline \multirow{4}{*}{ OsCPK3 } & \multirow{2}{*}{ GTTT } & 1 & NGT & $19 / 40(47.50 \%)$ & $19 / 19(100.00 \%)$ & $0 / 19(0.00 \%)$ & $36 / 40(90.00 \%)$ & \multirow{4}{*}{$600 \mathrm{bp}$} & \multirow{2}{*}{0} \\
\hline & & 2 & NGT & $8 / 40(20.00 \%)$ & $8 / 8(100.00 \%)$ & $0 / 8(0.00 \%)$ & $27 / 40(67.50 \%)$ & & \\
\hline & \multirow{2}{*}{ GCCC } & 1 & NGT & $5 / 30(16.67 \%\}$ & $5 / 5(100.00 \%)$ & $0 / 5(0.00 \%)$ & $30 / 30(100.00 \%)$ & & \multirow{2}{*}{0} \\
\hline & & 2 & NGT & $1 / 30(3.33 \%)$ & $1 / 1(100.00 \%)$ & $0 / 1(0.00 \%)$ & $30 / 30(100.00 \%)$ & & \\
\hline \multirow{4}{*}{ OsCPK4 } & \multirow{2}{*}{ GTTT } & 1 & NGC & $0 / 41(0.00 \%)$ & $0 / 0(0.00 \%)$ & $0 / 0(0.00 \%)$ & $0 / 41(0.00 \%)$ & \multirow{4}{*}{$243 b p$} & \multirow{2}{*}{0} \\
\hline & & 2 & NGC & $1 / 41(2.13 \%)$ & $1 / 1(100.00 \%)$ & $0 / 1(0.00 \%)$ & $13 / 41(31.71 \%)$ & & \\
\hline & \multirow{2}{*}{$\mathrm{GCCC}$} & 1 & NGC & $0 / 42(0.00 \%)$ & $0 / 0(0.00 \%)$ & $0 / 0(0.00 \%)$ & $30 / 42(71.43 \%)$ & & \\
\hline & & 2 & NGC & $0 / 42(0.00 \%)$ & $0 / 0(0.00 \%)$ & $0 / 0(0.00 \%)$ & $42 / 42(100.00 \%)$ & & 0 \\
\hline OМMPK & GTTT & 1 & NAG & $2 / 35(5.71 \%)$ & $2 / 2(100.00 \%)$ & $0 / 2(0.00 \%)$ & NA & $203 \mathrm{hm}$ & 0 \\
\hline USMPK & जाI & 2 & NAG & $7 / 35(20.00 \%)$ & $7 / 7(100.00 \%)$ & $0 / 7(0.00 \%)$ & NA & $203 \mathrm{bp}$ & 0 \\
\hline & & 1 & NAA & $17 / 51(33.33 \%)$ & $11 / 17(64.71 \%)$ & $6 / 17(35.29 \%)$ & NA & & \\
\hline OsMPK9 & GTTT & 2 & NAA & $0 / 51(0.00 \%)$ & $0 / 0(0.00 \%)$ & $0 / 0(0.00 \%)$ & NA & 5,251 bp & 0 \\
\hline & GTTT & 1 & NAT & $20 / 39(51.28 \%)$ & $20 / 20(100.00 \%)$ & $0 / 20(0.00 \%)$ & $16 / 39(41.03 \%)$ & & 0 \\
\hline OSМPK 10 & जाI & 2 & NAT & $17 / 39(43.58 \%)$ & $17 / 17(100.00 \%)$ & $0 / 17(0.00 \%)$ & $9 / 39(23.08 \%)$ & $3760 \mathrm{hn}$ & 0 \\
\hline OSMPK 70 & & 1 & NAT & $1 / 40(2.50 \%)$ & $1 / 1(100.00 \%)$ & $0 / 1(0.00 \%)$ & $33 / 40(82.50 \%)$ & $3, / 60 \mathrm{bp}$ & \\
\hline & GCCC & 2 & NAT & $6 / 40(15.00 \%)$ & $6 / 6(100.00 \%)$ & $0 / 6(0.00 \%)$ & $32 / 40(80.00 \%)$ & & 0 \\
\hline 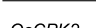 & OTTT & 1 & NAC & $14 / 47(29.79 \%)$ & $11 / 14(78.57 \%)$ & $3 / 14(21.43 \%)$ & NA & $270 \mathrm{~h}$ & $2 / 47$ \\
\hline OsCPK2 & GIII & 2 & NAC & $14 / 47(29.79 \%)$ & $12 / 14(85.71 \%)$ & $2 / 14(14.29 \%)$ & NA & $3 / 8 \mathrm{Dp}$ & $(4.26 \%)$ \\
\hline & CTTT & 1 & NTG & $0 / 31(0.00 \%)$ & $0 / 0(0.00 \%)$ & $0 / 0(0.00 \%)$ & $2 / 31(6.45 \%)$ & & $0_{0}>$ \\
\hline OSCPK14 & GाI & 2 & NTG & $0 / 31(0.00 \%)$ & $0 / 0(0.00 \%)$ & $0 / 0(0.00 \%)$ & $25 / 31(80.65 \%)$ & $318 \mathrm{hp}$ & 0 \\
\hline OSCFK 74 & $\operatorname{GCCC}$ & 1 & NTG & $0 / 38(0.00 \%)$ & $0 / 0(0.00 \%)$ & $0 / 0(0.00 \%)$ & $27 / 38(71.05 \%)$ & $318 \mathrm{bp}$ & 0 \\
\hline & $\mathrm{GCCC}$ & 2 & NTG & $0 / 38(0.00 \%)$ & $0 / 0(0.00 \%)$ & $0 / 0(0.00 \%)$ & $0 / 38(0.00 \%)$ & & 0 \\
\hline คח ח & OTTT & 1 & NTA & $0 / 34(0.00 \%)$ & $0 / 0(0.00 \%)$ & $0 / 0(0.00 \%)$ & NA & $181 \mathrm{hr}$ & 0 \\
\hline Oserno & דוा & 2 & NTA & $0 / 34(0.00 \%)$ & $0 / 0(0.00 \%)$ & $0 / 0(0.00 \%)$ & NA & 年 & 0 \\
\hline & & 1 & NTT & $0 / 31(0.00 \%)$ & $0 / 0(0.00 \%)$ & $0 / 0(0.00 \%)$ & $0 / 31(0.00 \%)$ & & \\
\hline OsCPK27 & GTTT & 2 & NTT & $0 / 31(0.00 \%)$ & $0 / 0(0.00 \%)$ & $0 / 0(0.00 \%)$ & $0 / 31(0.00 \%)$ & 327 bp & 0 \\
\hline & OTTT & 1 & NCG & $1 / 34(2.94 \%)$ & $1 / 1(100.00 \%)$ & $0 / 1(0.00 \%)$ & $23 / 34(67.65 \%)$ & & 0 \\
\hline ОЯРК Р & GIII & 2 & NCG & $0 / 34(0.00 \%)$ & $0 / 0(0.00 \%)$ & $0 / 0(0.00 \%)$ & $15 / 34(44.12 \%)$ & $849 \mathrm{hr}$ & 0 \\
\hline OSCPK 20 & scco & 1 & NCG & $0 / 38(0.00 \%)$ & $0 / 0(0.00 \%)$ & $0 / 0(0.00 \%)$ & $34 / 38(89.47 \%)$ & 849 bp & 0 \\
\hline & GCCC & 2 & NCG & $0 / 38(0.00 \%)$ & $0 / 0(0.00 \%)$ & $0 / 0(0.00 \%)$ & $32 / 38(84.21 \%)$ & & 0 \\
\hline O०PK21 & CTTT & 1 & NCA & $0 / 40(0.00 \%)$ & $0 / 0(0.00 \%)$ & $0 / 0(0.00 \%)$ & NA & $251 \mathrm{hr}$ & 0 \\
\hline USCPK 27 & जाI & 2 & NCA & $0 / 40(0.00 \%)$ & $0 / 0(0.00 \%)$ & $0 / 0(0.00 \%)$ & NA & $251 \mathrm{pp}$ & 0 \\
\hline & GTTT & 1 & NTC & $34 / 46(73.91 \%)$ & $30 / 34(88.24 \%)$ & $4 / 34(11.76 \%)$ & $28 / 46(60.87 \%)$ & & 0 \\
\hline OPRKOP & GTTT & 2 & $\mathrm{NCT}$ & $0 / 46(0.00 \%)$ & $0 / 0(0.00 \%)$ & $0 / 0(0.00 \%)$ & $36 / 46(78.26 \%)$ & $172 \mathrm{hr}$ & 0 \\
\hline OsCPK28 & scres & 1 & NTC & $3 / 37(7.50 \%)$ & $3 / 3(100.00 \%)$ & $0 / 3(0.00 \%)$ & $37 / 37(100.00 \%)$ & 172 bp & 0 \\
\hline & GeCl & 2 & NCT & $0 / 37(0.00 \%)$ & $0 / 0(0.00 \%)$ & $0 / 0(0.00 \%)$ & $36 / 37(97.30 \%)$ & & 0 \\
\hline OsMPK3 & GTTT & 1 & NCT & $2 / 47(4.26 \%)$ & $2 / 2(100.00 \%)$ & $0 / 2(0.00 \%)$ & NA & NA & 0 \\
\hline /OsMPK4 & GIII & 2 & NCC & $0 / 47(0.00 \%)$ & $0 / 0(0.00 \%)$ & $0 / 0(0.00 \%)$ & NA & NA & 0 \\
\hline OsMPK3 & GTTT & 1 & NTC & $0 / 52(0.00 \%)$ & $0 / 0(0.00 \%)$ & $0 / 0(0.00 \%)$ & NA & NA & 0 \\
\hline /OsMPK4 & GाI & 2 & NCC & $4 / 52(7.69 \%)$ & $4 / 4(100.00 \%)$ & $0 / 4(0.00 \%)$ & NA & NA & 0 \\
\hline 100 & & & & & & & & & \\
\hline 80 & & & & & On-target mutati & n frequency & Self-editing fre & uency of $s$ & RNA \\
\hline 60 & & & & & & & & & \\
\hline 40 & & & & & & & & & \\
\hline 20 & & & & & & & & & \\
\hline 0 & & & & In & 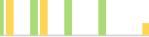 & & 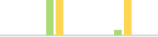 & & \\
\hline
\end{tabular}

Fig. 2 Analysis of SpRY nuclease activity on 16 possible NNN PAMs in transgenic rice. a Summary of the genotyping results on T0 transgenic rice lines generated with SpRY nuclease using different PAM sequences and sgRNA scaffolds. NA, not available. $\mathbf{b}$ Comparison of the on-target mutation efficiency and the selfediting frequency of sgRNA transgenes by SpRY nuclease toward different PAMs in T0 transgenic rice lines

more than $4.26 \%$ lines were detected with modified $\operatorname{sgRNAs}$ (Fig. 2a, b). On the other hand, mutation frequencies at the other high-efficiency $s g R N A$ sites (such as OsCPK3sgRNA1, OsCPK3-sgRNA2, OsMPK10-sgRNA1, OsCPK28-sgRNA1) were much higher, ranging from 23.08 to $100 \%$ (Fig. 2a, b). Also, varying self-editing frequencies (0$80.65 \%$ ) were observed in the low-efficiency $s g R N A$ s (i.e., OsCPK5-sgRNA2, OsCPK14sgRNA1, OsCPK14-sgRNA2, OsCPK27-sgRNA1) (Fig. 2a, b). It should be mentioned that 
on-target editing and self-editing of SpRY were detected simultaneously or alone in individual transgenic line, and DNA fragment deletion events between two sgRNAs in the T-DNA regions were detected in targeting OsCPK4 and OsCPK28, respectively (Additional file 1: Figures S6; S13). Combining all the data, we conclude that SpRY elicits complex self-editing events which might occur before and after gene editing of on-targets in transgenic rice.

Previous study has shown that utilizing a GCCC-type sgRNA scaffold alleviates the self-targeting property of SpCas9-NG without substantial loss of on-target activity in rice [41]. Therefore, we replaced the original GTTT-type sgRNA in our CRISPR/SpRY system with the GCCC-type sgRNA scaffold (Additional file 1: Table S1). Subsequently, we tested the nuclease activity of our modified CRISPR/SpRY system in transgenic rice callus using the $s g R N A s$ with high self-editing frequencies. To our surprise, replacing the GTTT-type scaffold with the GCCC-type scaffold did not show any improvement on on-target editing (Fig. 2a). On the contrary, the editing efficiency of OsCPK3, OsMPK10, and OsCPK28 was significantly reduced (Fig. 2a). We further investigated the identities of each sgRNA transgene in all lines and found that, out of 12 sgRNAs examined, eleven lines carried mutations at high frequencies (Fig. 2a). Based on our data, we conclude that the GCCC-type sgRNA scaffold elicits stronger self-editing events in sgRNA transgenes, which results in a decrease in the efficiency of on-target editing events in rice, as compared to the naturally occurring (original) GTTT-type sgRNA scaffold.

\section{SpRY mediates cytosine base editing at non-canonical PAM sites in the rice genome}

PAM preferences of Cas proteins largely limit the targeting scope of base editors [7, 8, 12, 32, 33]. Considering the greatly relaxed PAM compatibility of SpRY, we tested whether it could improve cytosine base editing in transgenic rice when fusing to the hyperactive cytidine deaminase hAID* $\Delta$. The SpRY nuclease was mutated into the SpRY(D10A) nickase and employed to replace the SpCas9n gene in the previously reported cytosine base editor rBE9 which enables C-to-D editing [7], resulting in rBE66 (Fig. 3a).

The base-editing activity of rBE66 was then investigated in transgenic rice callus with three sgRNAs targeting the endogenous OsCOI2 gene toward non-canonical NGC, NGT, and NAG PAMs, respectively. Intriguingly, of the 50 independent transgenic lines confirmed by sequencing, thirteen lines were identified with nucleotide mutations at the NGC PAM site which is presumably less efficient for SpRY targeting (Fig. 3b, e, h). Among these thirteen lines, twelve lines (24\% efficiency) carried nucleotide substitutions (predominantly the C-to-T conversion) in the typical editing window whereas six lines (12\% frequency) carried indels (Fig. 3b, e, h). These indels likely result from both the deaminase activity of hAID* $\Delta$ and the nickase activity of SpRY based on the position of indels. For the other two PAMs tested in OsCOI2, while the NGT PAM was inefficient, the 1 bp-shifted NAG PAM resulted in a base editing efficiency of 34.15\% (14 out of 41 lines) and indel frequency of $4.88 \%$ (2 out of 41 lines) (Fig. 3c, f, h). rBE66 was also tested with two sgRNAs targeting the endogenous $B S R-K 1$ gene at NGA and NAG PAM sites, respectively. After genotyping the transgenic lines obtained for both transformation constructs, 


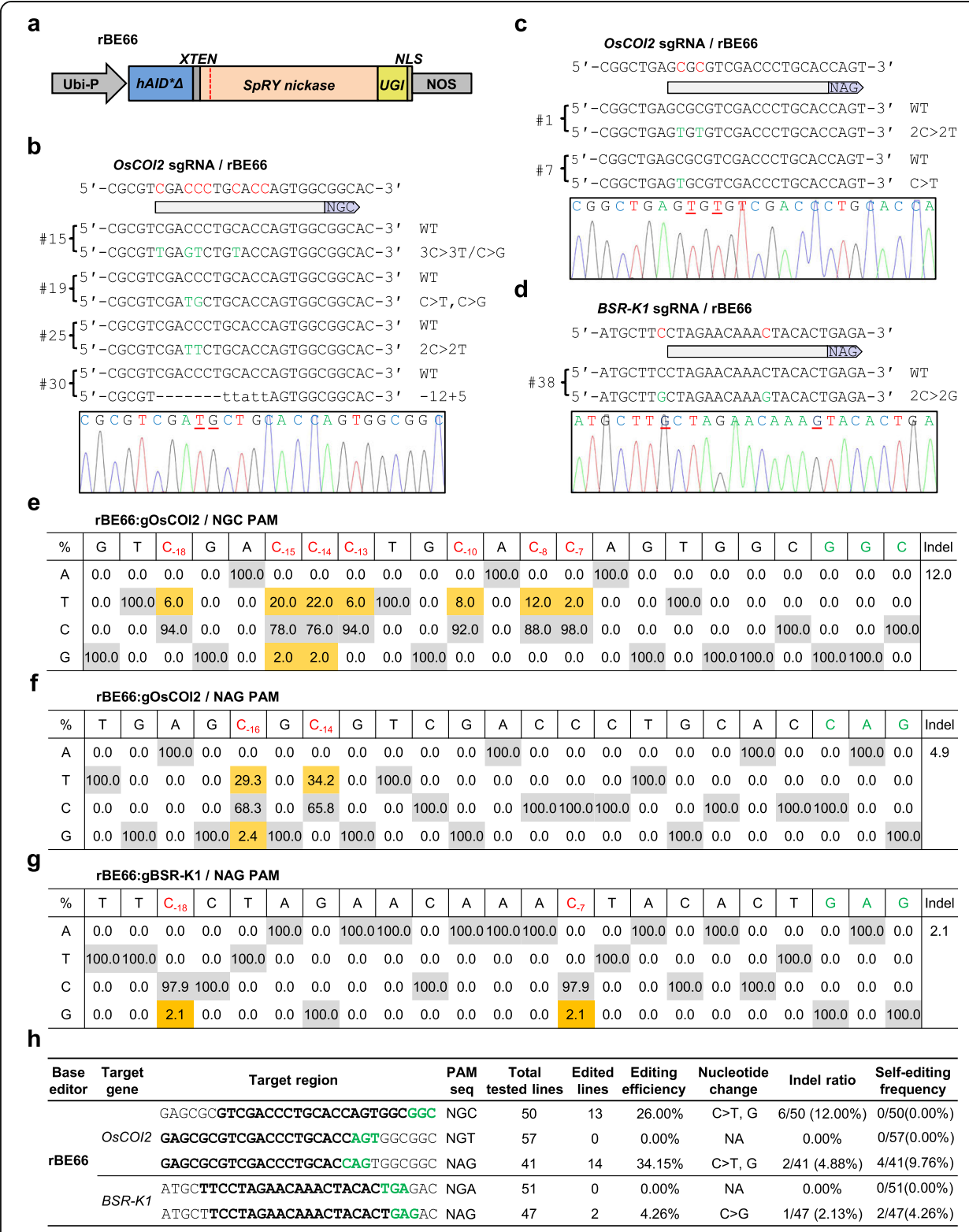

Fig. 3 Efficient cytosine base editing mediated by SpRY-fused hAID* $\Delta$ toward non-canonical PAMs in rice. a The gene construct of $r B E 66$ used for cytosine base editing in transgenic rice. Ubi-P, maize ubiquitin 1 promoter; $h A I D^{*} \Delta$, truncated version of hyperactive human AID gene; UGI, uracil glycosylase inhibitor; NLS, nuclear localization signal. b-d Representative edited alleles of OSCOI2 toward a NGC PAM (b) and a NAG PAM (c), and BSR-K1 toward a NAG PAM (d) generated by rBE66 in T0 transgenic rice lines. The PAM sequences for each target site are shown in the pale purple arrows; the candidate bases in the putative editing window for editing and detected nucleotide changes are highlighted in red and green, respectively; the nucleotide substitutions are underlined in the sequencing chromatograms. e- $\mathbf{g}$ Summary of nucleotide changes in the editing window of OsCOI2 toward a NGC PAM (e) and a NAG PAM (f), and BSR-K1 toward a NAG PAM (g) caused by rBE66 in T0 transgenic lines. The PAM sequences and the detected nucleotide changes are highlighted in green and red, respectively. $\mathbf{h}$ Summary of the genotyping results on T0 transgenic rice lines generated with rBE66. NA, not available

we only identified a single heterozygous line $(2.13 \%)$ with an atypical base editing event and a single heterozygous line $(2.13 \%)$ carrying indel at the NAG PAM site (Fig. 3d, g, h). Finally, the self-editing effect of rBE66 was also investigated. We identified 4 lines $(9.76 \%$ ratio) and 2 lines (4.26\% ratio) carrying nucleotide 
substitutions in OsCOI2-sgRNA (NAG PAM) and BSR-K1-sgRNA (NAG PAM) transgenes, respectively (Fig. $3 \mathrm{~h}$, Additional file 1: Figure S17). These data, combined, indicate that $S p R Y$ is compatible with hAID* $\Delta$, greatly expanding the targeting scope of hAID* $\Delta$-mediated cytosine base editor in rice. Thus, SpRY has very promising prospects in targeted base editing in plants due to the broad PAM compatibility and negligible self-editing effect.

SpRY-fused TadA8e monomer enables efficient adenine base editing at non-G PAM sites in the rice genome

The CRISPR/Cas-guided adenine deaminase heterodimer TadA: TadA7.10 is capable of introducing A-to-G conversion in rice [12, 32, 33]. Very recently, an evolved version TadA8e with a more robust enzymatic activity has been characterized in human cells $[14,40]$. Therefore, we investigated whether SpRY could improve adenine base editing in rice by fusing it to TadA8e. TadA7.10 carrying mutations A109S/T111R/D119N/H122N/Y147D/F149Y/T166I/D167N was rice codon-optimized (Additional file 1: Table S1) and fused to the 5 '-terminus of

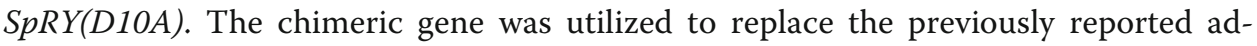
enine base editor gene rBE14 in the binary vector pUbi:rBE14 [12], resulting in pUbi:rBE62 (Fig. 4a).

The endogenous OsMPK13, OsGS1, BSR-D1, and OsGSK4 gene were utilized to test rBE62's activity toward the NAA, NAT, and NAC PAM in transgenic rice calli, respectively. Strikingly, rBE62 resulted in a base editing efficiency of $29.79 \%$ (14 out of 47 lines) at NAA PAM target site in OsMPK13, 93.75\% (45 out of 48 lines) at NAT PAM target site in OsGS1, 51.28\% (20 out of 39 lines) at NAC PAM target site in OsGSK4, and $0 \%$ at NAT PAM target site in BSR-D1, respectively (Fig. $4 \mathrm{~b}-\mathrm{h}$ ). All mutant lines carried mono-allelic mutations characterized by pure A-to-G conversion within an activity window (-17 to -9 positions upstream of PAM sequence), and no indel mutations were detected in the on-target regions (Fig. 4b-h). Furthermore, we genotyped the $s g R N A$ transgenes in all lines to evaluate the self-targeting effect of rBE62. Only one self-edited line $(2.13 \%$ ratio $)$ in which the $s g R N A$ transgene carried A-to-G conversion was detected. Nevertheless, no modification was observed in the target region since the entire OsMPK13 was intact (Additional file 1: Figure S17). These data, combined, indicate that the adenine base editor rBE62 (engineered with SpRY and TadA8e monomer as mentioned above) is capable of efficiently inducing A-to-G conversions in a wide editable range of the rice genome, including non-G PAM sites.

\section{Discussion}

PAM preference is the key limitation to each CRISPR-based tool for targeted genome editing, especially for base editing since it requires the precise positioning of Cas proteins at a given site. Therefore, reducing or eliminating the PAM requirement of Cas proteins will substantially advance various CRISPR technologies. To date, several other Cas proteins with altered or relaxed PAM specificities besides SpCas9, including SpCas9-VQR (NGA), SpCas9-VRER (NGA), SpCas9-NG (NG), ScCas9 (NNG), and SaKKH-Cas9 (NNNRRT), have been successfully adopted in both cytosine and/or 
adenine base editing in plants [8, 32-34]. The latest reported SpCas9 variants, SpG and SpRY with relaxed PAM specificity [39], might further optimize plant CRISPR tools for targeted base editing. However, different from the original report that SpG nuclease shows high-activity editing across all NGN PAM sites in human cells [39], we observed a different PAM preference (NGN/NAN/NTN>NCN) in transgenic rice lines. Moreover, our data also shows that the editing efficiency of SpG is lower than that of SpCas9-NG in transgenic rice lines. Therefore, we believe that SpCas9-NG is still the best genome editing player for NGN PAM sites in plants.

SpRY exhibits a similar preference for NGN PAM sequences as SpG. However, SpRY nuclease is also capable of processing many NAN, NTC, NCT, NCC, and NCG PAM sites with varying efficiency, which have previously been inaccessible

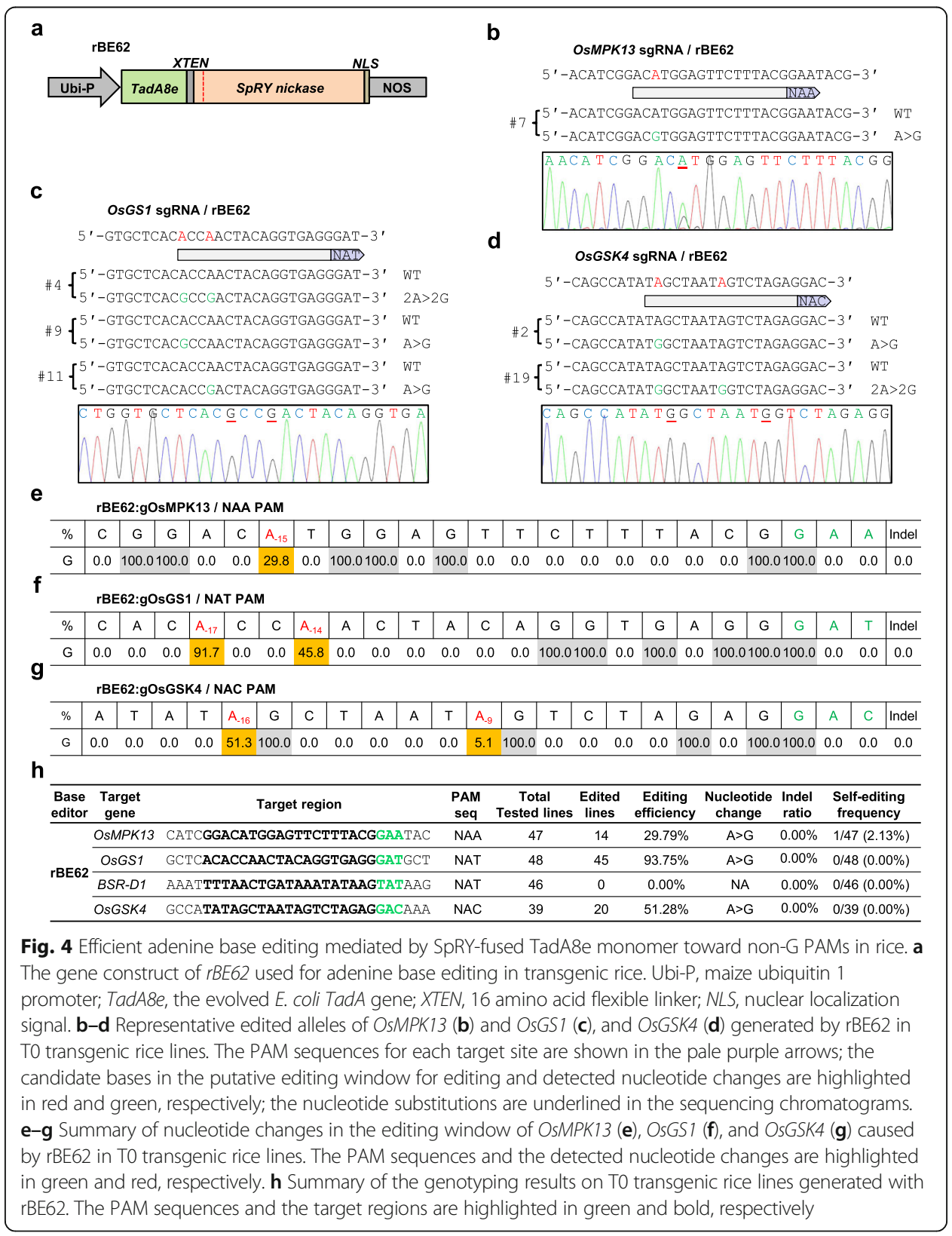


with CRISPR tools. SpRY thereby greatly increases the number of targeting sites previously not available for genome editing in rice. However, as shown in our study, the sgRNA-editing frequencies were extremely high, whereas the on-target editing frequencies were very low (even $0 \%$ efficiency) in some cases. We assumed that disruption of sgRNA sequences by self-editing has prevented on-target from editing. Therefore, the broadened PAM compatibility of SpRY nuclease can result in complex and unpredictable self-targeting of sgRNA transgenes, which presumably affects the on-target editing at both early- and late-transformation stages. Nevertheless, we show that the naturally occurring GTTT-type of guide RNA scaffold outperforms the modified GCCC-type [41] when using SpRY to edit some endogenous genomic sites, and we do not recommend SpRY for multiple gene knocking out since it causes loss of sgRNA transgenes in some cases. More GTNand GCN-type sgRNA scaffolds in self-editing and the heritability of edited genes in generations should be investigated in the future.

The SpRY nickase was compatible with both hAID* $\Delta$ and TadA8e deaminases and achieved efficient cytosine and adenine base editing at various non-canonical PAM sites, especially non-G PAM sites. Intriguingly, compared to the highfrequency self-editing caused by the SpRY nuclease in transgenic rice, significantly low ratio of self-editing of $s g R N A$ transgenes was detected in our base editing assay, in which mutations are the result of the activity of the TadA8e deaminase and not the SpRY nickase. In our study, we also show that the newly evolved TadA8e, as reported in human cells [14], enables efficient A-to-G conversion in rice as well. Therefore, both SpRY and Tad8e can be presumably utilized to multiplex base editing in rice and further optimize other plant base editors in terms of targeting scope and editing efficiency.

The broadened PAM compatibility of SpRY raises the possibility of increased offtarget editing across all plant genomes. To address this question, the fidelity-enhancing substitutions of SpCas9-HF1 could be introduced into the SpRY nuclease to alleviate its off-target effect in rice as reported in human cells [39]. Alternatively, careful design of the sgRNA is another way to avoid off-target cleavage. For example, off-target mutations were detected only for $s g R N A s$ targeting OsCPK3 and OsCPK28 in our study (Additional file 1: Figure S18). Regarding the SpRY nickase-mediated base editing with specific sgRNAs, hAID* $\Delta$ and TadA8e-dependent off-target base editing are the major issues, which might be minimized through protein engineering in the future. Nevertheless, timely isolation of the transgene-free, gene-edited lines in T1 progenies to prevent continual editing is highly recommended, and backcrossing it with the recipient material to purify the genetic background can be carried out if needed.

\section{Conclusions}

The structurally engineered SpRY efficiently recognizes NAN in addition to NGN PAM sequences, greatly expanding the targetable range of the rice genome. With the new SpRY-based tools added into the toolbox, plant biologists can easily carry out highresolution genome editing now, and we recommend using SpCas9 for target sites carrying NGG PAMs, SpCas9-NG for NGH PAMs (where H is A, C, or T), ScCas9 for NHG PAMs, and SpRY for NAH PAMs. 


\section{Methods}

\section{Rice materials}

Rice cultivars Kitaake (Oryza sativa L. ssp. Geng) was used in this study and kept in our lab. Rice plants were cultivated in the paddy field under natural conditions in normal rice growing seasons, and immature seeds were harvested from expanded panicles for use in rice transformation.

\section{Vector design and plasmid construction}

The coding regions of $S p G$ gene [39] flanked by two nuclear localization signal (NLS) sequences were rice codon-optimized, and synthesized separately ( $S p G-f g 1$ and $S p G$ $f g 2$ ) by Tsingke (Beijing) (Additional file 1: Table S1). The full-length $S p G$ was assembled using the overlapping-extension PCR-based method with the high-fidelity DNA polymerase Phusion (NEB) and the primers listed in Additional file 1: Table S3, and directly cloned downstream of the maize ubiquitin 1 promoter and upstream of the NOS terminator by replacing the Cas9-NG gene in pUbi:Cas9-NG [32] through BamHI/SpeI digestion and DNA ligation, resulting in the binary vector pUbi:SpG.

For the construction of the binary vector of SpRY nuclease, a point mutation of GCG to AGG (A61R) was first introduced into $S p G-f g 1$ by PCR amplification of whole plasmid pUC57:SpG-fg1 with the primer pairs SpRY-F1/SpRY-R1. The amplicon was self-ligated and confirmed by sequencing, resulting in pUC57:SpRY-fg1. Next, the 3' region of $\operatorname{SpRY}$ [39] fused with an NLS sequence was rice codon-optimized (SpRY-fg2) and subjected to synthesizing by Tsingke (Beijing) (Additional file 1: Table S1). Finally, both $S p R Y-f g 1$ and $S p R Y-f g 2$ were assembled and pUbi:SpRY was constructed in the same manner as mentioned for $S p G$.

For the construction of cytosine base editors, the gene fusion of $h A I D * \Delta, S p R Y$, and UGI fragment was carried out with a simple PCR-based cloning strategy. Briefly, the full-length $\operatorname{SpRY}$ gene was PCR amplified using the primer pairs OsCas9-Fg1-F1/ OsCas9-Fg1-R1 and pUbi:SpRY as the template, and ligated with the backbone of pUC19:rBE9 [7] carrying both $h A I D * \Delta$ and UGI fragment, which was amplified with the primer pairs UGI-F1/rAPO-R1, resulting in pUC19:rBE66. For the construction of adenine base editors, the TadA8e fragment was codon-optimized and subjected to synthesizing by Tsingke (Beijing) (Additional file 1: Table S1), and fused to the $5^{\prime}$ end of $\operatorname{SpR} Y$ using the overlapping-extension PCR-based method with the primers listed in Table S3, resulting in rBE62 fragment. Finally, rBE66 and rBE62 were cloned into the binary vector by BamHI/SpeI digestion as described above, resulting in pUbi:rBE66 for cytosine base editing and pUbi:rBE62 for adenine base editing in rice, respectively.

For the construction of guide RNA gene, the GCCC-type sgRNA [41] was subjected to synthesizing by Tsingke (Beijing) (Additional file 1: Table S1), amplified with the primer pairs gRNA4-NG-F2/gRNA4-NG-R2, and used to replace the GTTT-type sgRNA in pENTR4:sgRNA4 using In-Fusion HD Enzyme Premix (Clontech), resulting in the entry vector pENTR4:sgRNA4-NG.

For the construction of the final T-DNA transformation plasmids, the 19 20-bp complementary oligos initialized by "G" (Additional file 1: Table S2), corresponding to each target site (Additional file 1: Table S3) and carrying appropriate 4-bp adaptor, were phosphorylated, annealed, and inserted into the BsaI-digested pENTR4:sgRNA4 or pENTR4: 
sgRNA4-NG. Each sgRNA expression cassette was then shuffled into the respective binary vector by LR clonase (Invitrogen). All primers used here are listed in Table S3.

The identities of $S p G, S p R Y, r B E 66$, and $r B E 62$ in vectors and oligo insertions in each construct were confirmed by Sanger sequencing.

\section{Agrobacterium-mediated rice transformation}

T-DNA transformation plasmids harboring gene-targeting sgRNA were transferred into the A. tumefaciens strain EHA105 by electroporation. Rice callus derived from immature seeds of Kitake was used for stable transformation following a protocol as described previously [28].

\section{Genotyping of transgenic rice lines}

Genomic DNA was isolated from each T0 transgenic callus line using the hexadecyltrimethylammonium bromide (CTAB) method [42]. PCR amplification of the targeted genomic region was carried out with specific primers listed in Additional file 1: Table S3, and the PCR products were subjected to Sanger sequencing. Besides, a selection of PCR products with low quality of sequencing data was cloned into the pEASY-Blunt cloning vector (TransGen Biotech), and colonies with insertion were randomly chosen for Sanger sequencing.

\section{Supplementary Information}

The online version contains supplementary material available at https://doi.org/10.1186/s13059-020-02231-9.

Additional file 1. Supplemental figures 1-18 and Supplementary Tables 1-3.

Additional file 2. Review history.

Acknowledgements

We thank Sujie Zhang and Xueqi Li for assistance with tissue culture of rice and DNA manipulation.

Review history

The review history is available as Additional file 2.

Peer review information

Kevin Pang was the primary editor of this manuscript and managed its editorial process and peer review in collaboration with the rest of the editorial team.

Authors' contributions

$H Z, X Z, G W, C S, W S$, and BR designed the research; ZX, YK, BR, DY, and FY conducted the experiments and analyzed the data; $H Z$, XZ, and GW supervised the research; $H Z, X Z$, and CS wrote the original draft; all authors participated in the discussion and revision of the manuscript. The authors read and approved the final manuscript.

Funding

This study was supported by grants from the National Natural Science Foundation of China (31871948), the Fundamental Research Funds, and the Agricultural Science and Technology Innovation Program of the Chinese Academy of Agricultural Sciences (Y2020PT26) to HZ; the National Transgenic Science and Technology Program of China (2019ZX08010-003) to FY; and the National Natural Science Foundation of China (31930089) to XZ.

Availability of data and materials

All data generated in the study has been included in the manuscript and additional files.

Ethics approval and consent to participate

Not applicable.

Competing interests

The authors have filed a patent application based on the results reported in this study. 


\section{Author details}

'State Key Laboratory for Biology of Plant Diseases and Insect Pests, Institute of Plant Protection, Chinese Academy of Agricultural Sciences, Beijing 100193, China. ${ }^{2}$ Scientific Observing and Experimental Station of Crop Pests in Guilin, Ministry of Agriculture and Rural Affairs, Guilin 541399, China. ${ }^{3}$ Shenzhen Branch, Guangdong Laboratory for Lingnan Modern Agriculture, Genome Analysis Laboratory of the Ministry of Agriculture, Agricultural Genomics Institute at Shenzhen, Chinese Academy of Agricultural Sciences, Shenzhen 518120, China. ${ }^{4}$ College of Plant Protection, China Agricultural University, Beijing 100193, China. ${ }^{5}$ Norwegian Institute of Bioeconomy Research, 1432 Aas, Norway. ${ }^{6}$ College of Plant Protection, Jilin Agricultural University, Changchun 130118, China. ${ }^{7}$ State Key Laboratory of Rice Biology, Institute of Biotechnology, Zhejiang University, Hangzhou 310058, China.

Received: 12 September 2020 Accepted: 10 December 2020

Published online: 04 January 2021

\section{References}

1. Hille F, Richter H, Wong SP, Bratovic M, Ressel S, Charpentier E. The biology of CRISPR-Cas: backward and forward. Cell. 2018;172:1239-59.

2. Zhou H, Liu B, Weeks DP, Spalding MH, Yang B. Large chromosomal deletions and heritable small genetic changes induced by CRISPR/Cas9 in rice. Nucleic Acids Res. 2014;42:10903-14.

3. Li J, Meng X, Zong Y, Chen K, Zhang H, Liu J, Li J, Gao C. Gene replacements and insertions in rice by intron targeting using CRISPR-Cas9. Nat Plants. 2016;2:16139.

4. Lu Y, Tian Y, Shen R, Yao Q, Wang M, Chen M, Dong J, Zhang T, Li F, Lei M, Zhu J-K. Targeted, efficient sequence insertion and replacement in rice. Nat Biotechnol. 2020. https://doi.org/10.1038/s41587-020-0581-5.

5. Tan J, Zhao Y, Wang B, Hao Y, Wang Y, Li Y, Luo W, Zong W, Li G, Chen S, et al. Efficient CRISPR/Cas9-based plant genomic fragment deletions by microhomology-mediated end joining. Plant Biotechnol J. 2020. https://doi.org/10.1111/ pbi.13390.

6. Sun Y, Zhang X, Wu C, He Y, Ma Y, Hou H, Guo X, Du W, Zhao Y, Xia L. Engineering herbicide-resistant rice plants through CRISPR/Cas9-mediated homologous recombination of acetolactate synthase. Mol Plant. 2016;9:628-31.

7. Ren B, Yan F, Kuang Y, Li N, Zhang D, Zhou X, Lin H, Zhou H. Improved base editor for efficiently inducing genetic variations in rice with CRISPR/Cas9-guided hyperactive hAID mutant. Mol Plant. 2018;11:623-6.

8. Ren B, Yan F, Kuang Y, Li N, Zhang D, Lin H, Zhou H. A CRISPR/Cas9 toolkit for efficient targeted base editing to induce genetic variations in rice. Sci China Life Sci. 2017;60:516-9.

9. Li C, Zong Y, Wang Y, Jin S, Zhang D, Song Q, Zhang R, Gao C. Expanded base editing in rice and wheat using a Cas9adenosine deaminase fusion. Genome Biol. 2018;19:59.

10. Zhao D, Li J, Li S, Xin X, Hu M, Price MA, Rosser SJ, Bi C, Zhang X. Glycosylase base editors enable C-to-A and C-to-G base changes. Nat Biotechnol. 2020. https://doi.org/10.1038/s41587-020-0592-2.

11. Kurt IC, Zhou R, lyer S, Garcia SP, Miller BR, Langner LM, Grunewald J, Joung JK. CRISPR C-to-G base editors for inducing targeted DNA transversions in human cells. Nat Biotechnol. 2020. https://doi.org/10.1038/s41587-020-0609-X.

12. Yan F, Kuang Y, Ren B, Wang J, Zhang D, Lin H, Yang B, Zhou X, Zhou H. Highly efficient A.T to G.C base editing by Cas9n-guided tRNA adenosine deaminase in rice. Mol Plant. 2018;11:631-4.

13. Gaudelli NM, Lam DK, Rees HA, Sola-Esteves NM, Barrera LA, Born DA, Edwards A, Gehrke JM, Lee SJ, Liquori AJ, et al. Directed evolution of adenine base editors with increased activity and therapeutic application. Nat Biotechnol. 2020;38: 892-900.

14. Richter MF, Zhao KT, Eton E, Lapinaite A, Newby GA, Thuronyi BW, Wilson C, Koblan LW, Zeng J, Bauer DE, et al. Phageassisted evolution of an adenine base editor with improved Cas domain compatibility and activity. Nat Biotechnol. 2020; 38:883-91.

15. Grunewald J, Zhou R, Lareau CA, Garcia SP, lyer S, Miller BR, Langner LM, Hsu JY, Aryee MJ, Joung JK. A dual-deaminase CRISPR base editor enables concurrent adenine and cytosine editing. Nat Biotechnol. 2020;38:861-4.

16. Li C, Zhang R, Meng X, Chen S, Zong Y, Lu C, Qiu JL, Chen YH, Li J, Gao C. Targeted, random mutagenesis of plant genes with dual cytosine and adenine base editors. Nat Biotechnol. 2020;38:875-82.

17. Zhang X, Zhu B, Chen L, Xie L, Yu W, Wang Y, Li L, Yin S, Yang L, Hu H, et al. Dual base editor catalyzes both cytosine and adenine base conversions in human cells. Nat Biotechnol. 2020;38:856-60.

18. Lin Q, Zong Y, Xue C, Wang S, Jin S, Zhu Z, Wang Y, Anzalone AV, Raguram A, Doman JL, et al. Prime genome editing in rice and wheat. Nat Biotechnol. 2020;38:582-5.

19. Li H, Li J, Chen J, Yan L, Xia L. Precise modifications of both exogenous and endogenous genes in rice by prime editing. Mol Plant. 2020;13:671-4.

20. Tang X, Sretenovic S, Ren Q, Jia X, Li M, Fan T, Yin D, Xiang S, Guo Y, Liu L, et al. Plant prime editors enable precise gene editing in rice cells. Mol Plant. 2020;13:667-70.

21. Xu R, Li J, Liu X, Shan T, Qin R, Wei P. Development of plant prime-editing systems for precise genome editing. Plant Commun. 2020;1:100043.

22. Hsu PD, Scott DA, Weinstein JA, Ran FA, Konermann S, Agarwala V, Li Y, Fine EJ, Wu X, Shalem O, et al. DNA targeting specificity of RNA-guided Cas9 nucleases. Nat Biotechnol. 2013;31:827-32

23. Sternberg SH, Redding S, Jinek M, Greene EC, Doudna JA. DNA interrogation by the CRISPR RNA-guided endonuclease Cas9. Nature. 2014;507:62-7.

24. Li Z, Liu ZB, Xing A, Moon BP, Koellhoffer JP, Huang L, Ward RT, Clifton E, Falco SC, Cigan AM. Cas9-guide RNA directed genome editing in soybean. Plant Physiol. 2015;169:960-70

25. Lawrenson T, Shorinola O, Stacey N, Li CD, Ostergaard L, Patron N, Uauy C, Harwood W. Induction of targeted, heritable mutations in barley and Brassica oleracea using RNA-guided Cas9 nuclease. Genome Biol. 2015;16:258.

26. Barone P, Wu E, Lenderts B, Anand A, Gordon-Kamm W, Svitashev S, Kumar S. Efficient gene targeting in maize using inducible CRISPR-Cas9 and marker-free donor template. Mol Plant. 2020;13:1219-27.

27. Gosavi G, Yan F, Ren B, Kuang Y, Yan D, Zhou X, Zhou H. Applications of CRISPR technology in studying plant-pathogen interactions: overview and perspective. Phytopathol Res. 2020;2:21. 
28. Kuang Y, Li S, Ren B, Yan F, Spetz C, Li X, Zhou X, Zhou H. Base-editing-mediated artificial evolution of OSALS1 in planta to develop novel herbicide-tolerant rice germplasms. Mol Plant. 2020;13:565-72.

29. Kleinstiver BP, Prew MS, Tsai SQ, Topkar W, Nguyen NT, Zheng Z, Gonzales AP, Li Z, Peterson RT, Yeh JR, et al. Engineered CRISPR-Cas9 nucleases with altered PAM specificities. Nature. 2015;523:481-5.

30. Hu JH, Miller SM, Geurts MH, Tang W, Chen L, Sun N, Zeina CM, Gao X, Rees HA, Lin Z, Liu DR. Evolved Cas9 variants with broad PAM compatibility and high DNA specificity. Nature. 2018;556:57-63.

31. Nishimasu H, Shi X, Ishiguro S, Gao L, Hirano S, Okazaki S, Noda T, Abudayyeh OO, Gootenberg JS, Mori H, et al. Engineered CRISPR-Cas9 nuclease with expanded targeting space. Science. 2018;361:1259-62.

32. Ren B, Liu L, Li S, Kuang Y, Wang J, Zhang D, Zhou X, Lin H, Zhou H. Cas9-NG greatly expands the targeting scope of the genome-editing toolkit by recognizing NG and other atypical PAMs in rice. Mol Plant. 2019;12:1015-26.

33. Wang M, Xu Z, Gosavi G, Ren B, Cao Y, Kuang Y, Zhou C, Spetz C, Yan F, Zhou X, Zhou H. Targeted base editing in rice with CRISPR/ScCas9 system. Plant Biotechnol J. 2020;18:1645-7.

34. Hua K, Tao X, Zhu JK. Expanding the base editing scope in rice by using Cas9 variants. Plant Biotechnol J. 2019; 17:499-504.

35. Steinert J, Schiml S, Fauser F, Puchta H. Highly efficient heritable plant genome engineering using Cas9 orthologues from Streptococcus thermophilus and Staphylococcus aureus. Plant J. 2015;84:1295-305.

36. Karvelis T, Gasiunas G, Young J, Bigelyte G, Silanskas A, Cigan M, Siksnys V. Rapid characterization of CRISPR-Cas9 protospacer adjacent motif sequence elements. Genome Biol. 2015;16:253.

37. Tang X, Lowder LG, Zhang T, Malzahn AA, Zheng X, Voytas DF, Zhong Z, Chen Y, Ren Q, Li Q, et al. A CRISPR-Cpf1 system for efficient genome editing and transcriptional repression in plants. Nat Plants. 2017;3:17103.

38. Ming M, Ren Q, Pan C, He Y, Zhang Y, Liu S, Zhong Z, Wang J, Malzahn AA, Wu J, et al. CRISPR-Cas12b enables efficient plant genome engineering. Nat Plants. 2020;6:202-8.

39. Walton RT, Christie KA, Whittaker MN, Kleinstiver BP. Unconstrained genome targeting with near-PAMless engineered CRISPR-Cas9 variants. Science. 2020;368:290-6.

40. Lapinaite A, Knott GJ, Palumbo CM, Lin-Shiao E, Richter MF, Zhao KT, Beal PA, Liu DR, Doudna JA. DNA capture by a CRISPR-Cas9-quided adenine base editor. Science. 2020;369:566-71.

41. Qin R, Li J, Liu X, Xu R, Yang J, Wei P. SpCas9-NG self-targets the sgRNA sequence in plant genome editing. Nat Plants. 2020:6:197-201.

42. Porebski S, Bailey LG, Baum BR. Modification of a CTAB DNA extraction protocol for plants containing high polysaccharide and polyphenol components. Plant Mol Biol Report. 1997;15:8-15.

Publisher's Note

Springer Nature remains neutral with regard to jurisdictional claims in published maps and institutional affiliations.

Ready to submit your research? Choose BMC and benefit from:

- fast, convenient online submission

- thorough peer review by experienced researchers in your field

- rapid publication on acceptance

- support for research data, including large and complex data types

- gold Open Access which fosters wider collaboration and increased citations

- maximum visibility for your research: over $100 \mathrm{M}$ website views per year

At BMC, research is always in progress.

Learn more biomedcentral.com/submissions 